\title{
Flora arbustiva-arbórea de três áreas ribeirinhas no semiárido paraibano, Brasil
}

\author{
Alecksandra Vieira de Lacerda ${ }^{1,5}$, Francisca Maria Barbosa ${ }^{2}$, \\ João Juares Soares ${ }^{3}$ \& Maria Regina de Vasconcellos Barbosa ${ }^{4}$ \\ ${ }^{1}$ Centro de Desenvolvimento Sustentável do Semiárido, Unidade Acadêmica de Tecnologia do \\ Desenvolvimento, Universidade Federal de Campina Grande - UFCG, CEP 58540-000, Sumé, PB, Brasil \\ ${ }^{2}$ Instituto Nacional do Semiárido, Av. Floriano Peixoto, n. 715, Centro, \\ CEP 58100-001, Campina Grande, PB, Brasil \\ ${ }^{3}$ Centro de Ciências Biológicas e da Saúde, Departamento de Botânica, \\ Universidade Federal de São Carlos - UFSCar, CP 676, CEP 13565-905, São Carlos, SP, Brasil \\ ${ }^{4}$ Departamento de Sistemática e Ecologia, Universidade Federal da Paraíba - UFPB, CP 5065, \\ Cidade Universitária, CEP 58051-970, João Pessoa, PB, Brasil \\ ${ }^{5}$ Autor para correspondência: Alecksandra Vieira de Lacerda, e-mail: alecvieira@ufcg.edu.br
}

LACERDA, A.V., BARBOSA, F.M., SOARES, J.J. \& BARBOSA, M.R.V. Floristic composition of shrubbyarboreal component in three riparian vegetation areas in the State of Paraíba, semiarid region, Brazil. Biota Neotrop. 10(4): http://www.biotaneotropica.org.br/v10n4/en/abstract?inventory+bn01810042010.

\begin{abstract}
Aimed in the present work to study the floristic composition of shrubby and tree in three riparian vegetation areas and compare with other study realized in the basin of the Taperoá River, in the paraiban semiarid. A floristic survey was carried out from January 2004 throughout June 2006, covering riparian areas along the intermittent streams Cazuzinha ( $7^{\circ} 26^{\prime} 13^{\prime \prime} \mathrm{S}$ and $36^{\circ} 54^{\prime} 30^{\prime \prime} \mathrm{W}$; $564-579 \mathrm{~m}$ of altitude), Mares ( $7^{\circ} 31^{\prime} 53^{\prime \prime} \mathrm{S}$ and $36^{\circ} 33^{\prime} 39^{\prime \prime}$ W; 536-550 m of altitude), and Farias ( $7^{\circ} 25^{\prime} 33^{\prime \prime} \mathrm{S}$ and $36^{\circ} 29^{\prime} 21^{\prime \prime} \mathrm{W}$; 454-470 $\mathrm{m}$ of altitude). The floristic survey carried out in all three areas, generated a total of 91 species, of which, 68 were collected along Cazuzinha stream, 62 along Mares stream, and 56 along Farias stream. The families with the largest number of species and genera identified were: Fabaceae, Euphorbiaceae and Rubiaceae. Of the total of species registered for the three areas, 15 are present in the known lists of riverine bushes in other states of Brazil, 26 are endemic of the Caatinga (Brazilian northeastern savanna) and 10 of them had been exclusive of the researched area. The analysis of floristic similarity of the riparian areas of the present study in a comparison with other five surveys performed formerly showed that the highest floristic identity is related mainly to geographical distance and to the soil use and land occupancy.

Keywords: riparian forest, intermittent streams, floristic composition, species richness, caatinga.
\end{abstract}

LACERDA, A.V., BARBOSA, F.M., SOARES, J.J. \& BARBOSA, M.R.V. Flora arbustiva-arbórea de três áreas ribeirinhas no semiárido paraibano, Brasil. Biota Neotrop. 10(4): http://www.biotaneotropica.org.br/ v10n4/pt/abstract?inventory+bn01810042010.

Resumo: Objetivou-se no presente trabalho contribuir para o conhecimento florístico de três áreas ribeirinhas na caatinga e realizar a comparação destas com outros estudos desenvolvidos na bacia do Rio Taperoá, semiárido paraibano. O levantamento florístico foi realizado através de coletas assistemáticas no período de janeiro/2004 a junho/2006 e abrangeu as áreas ribeirinhas presentes ao longo dos riachos intermitentes do Cazuzinha ( $7^{\circ} 26^{\prime} 13^{\prime \prime} \mathrm{S}$ e $36^{\circ} 54^{\prime} 30^{\prime \prime} \mathrm{W}$; $564-579 \mathrm{~m}$ de altitude), Mares (7 $31^{\prime} 53^{\prime \prime} \mathrm{S}$ e $36^{\circ} 33^{\prime} 39^{\prime \prime} \mathrm{W}$; $536-550 \mathrm{~m}$ de altitude) e Farias ( $7^{\circ} 25^{\prime} 33^{\prime \prime} \mathrm{S}$ e $36^{\circ} 29^{\prime} 21^{\prime \prime} \mathrm{W} ; 454-470 \mathrm{~m}$ de altitude). O levantamento das três áreas registrou um total de 91 espécies, das quais 68 ocorreram na área do riacho do Cazuzinha, 62 no riacho dos Mares e 56 no riacho do Farias. As famílias com maior número de espécies e de gêneros foram Fabaceae, Euphorbiaceae e Rubiaceae. Do total de espécies registrado para as três áreas, 15 estão presentes nas listas relacionadas para matas ciliares de outros estados do Brasil, 26 são endêmicas da Caatinga e 10 foram exclusivas. Analisando a similaridade florística entre as áreas estudadas e outros cinco levantamentos, observou-se que a maior identidade florística está relacionada principalmente com a distância geográfica e às características de uso e ocupação da terra.

Palavras-chave: floresta ripária, riachos intermitentes, composição florística, riqueza de espécies, caatinga. 


\section{Introdução}

As áreas ribeirinhas apresentam formações vegetais características que se encontram associadas aos corpos d'água (Oliveira-Filho 1994) e podem se estender por dezenas de metros a partir das margens dos mesmos, apresentando variações na composição florística e na estrutura comunitária, dependendo das interações que estabelecem entre o ecossistema aquático e o ambiente terrestre adjacente. Estas formações são importantes no que tange aos recursos genéticos, florísticos, hídricos, edáficos (Santos \& Sousa-Silva 1998) e exercem destacado papel como corredores de fluxo gênico tanto vegetal quanto animal (Marinho-Filho \& Gastal 2004).

A conservação das áreas ciliares é, sem dúvida, inquestionável e sua importância ecológica vem fazendo com que muitos países elaborem instrumentos jurídicos visando a sua manutenção. No Brasil, o Código Florestal estabelece faixas de proteção variáveis em função da largura dos corpos d'água correspondentes. Entretanto, apesar das evidências de sua importância e de sua proteção legal, a cobertura vegetal presente nas áreas ciliares vem sendo constantemente degradada (Mueller 1998, van den Berg \& Oliveira-Filho 2000).

Quadros como o delineado acima são também evidentes em áreas de caatinga do semiárido paraibano. Neste estado a caatinga é o principal ecossistema, ocupando $40.539 \mathrm{~km}^{2}(71,64 \%)$ do território total (Paraíba 1992). Dentre as peculiaridades marcantes desse ecossistema, as matas ribeirinhas que recobrem as margens aluviais dos rios intermitentes que cortam o semiárido, são definidas como ambientes de exceção por apresentarem um padrão fisionômico e florístico diferenciado em relação a outras áreas na caatinga. Todavia, a exemplo do que vem ocorrendo em outras regiões do Brasil, essas matas na Paraíba também vêm sendo degradadas por apresentarem os solos mais adequados à agricultura (Paraíba 2005). Diante desse quadro de degradação da vegetação ribeirinha no semiárido paraibano e da crescente preocupação com a manutenção das funções ecológicas por elas desempenhadas, fica evidenciada a necessidade de iniciativas voltadas para sua conservação ou recuperação. Autores como van den Berg \& Oliveira-Filho (2000) têm apontado que estudos detalhados sobre a composição florística e a ecologia dos remanescentes dessas florestas são fundamentais para embasar quaisquer iniciativas para proteger, enriquecer, recuperar ou reconstituir esse tipo de vegetação. Desta forma, tem-se ratificado a relevância de trabalhos que busquem preencher grandes lacunas sobre o conhecimento e a quantificação de espécies típicas de áreas ribeirinhas da caatinga.

Este trabalho objetivou contribuir para o conhecimento florístico de três áreas ribeirinhas na caatinga e realizar a comparação destas com estudos desenvolvidos na bacia do Rio Taperoá e com outros levantamentos realizados nos ecossistemas ciliares brasileiros. Espera-se que os resultados obtidos possam subsidiar a conservação dessas áreas e servir de indicativos para modelos de enriquecimento e recuperação dos ambientes já degradados.

Este trabalho objetivou contribuir para o conhecimento florístico de três áreas ribeirinhas na caatinga e realizar a comparação destas com outros estudos desenvolvidos na bacia do Rio Taperoá, semiárido paraibano. Espera-se que os resultados obtidos possam subsidiar a conservação dessas áreas e servir de indicativos para modelos de enriquecimento e recuperação dos ambientes já degradados.

\section{Material e Métodos}

\section{1. Áreas de estudo}

A bacia hidrográfica do Rio Taperoá, no semiárido paraibano, drena uma área aproximada de $7.316 \mathrm{~km}^{2}$ (Paraíba 1997) e se localiza na parte central do Estado da Paraíba, entre as latitudes 6 $6^{\circ} 51$ ' 31 ' e $7^{\circ} 34^{\prime} 21^{\prime}$ 'S e entre as longitudes $36^{\circ} 0$ ' 55' e $37^{\circ} 13^{\prime}$ ' ' 'W (Lacerda
2003). As áreas ribeirinhas amostradas estão distribuídas ao longo dos riachos intermitentes do Cazuzinha, Mares e Farias (Figura 1), descritos a seguir com suas respectivas peculiaridades.

Riacho do Cazuzinha - localizado na sub-bacia do riacho dos Cordeiros, o Cazuzinha possui $15 \mathrm{~km}$ de extensão e bacia de drenagem de $59 \mathrm{~km}^{2}$. A área ribeirinha amostrada neste riacho (3,6 ha) está localizada na Reserva Particular do Patrimônio Natural (RPPN) Fazenda Almas, município de São José dos Cordeiros, entre as latitudes $7^{\circ} 26^{\prime} 13$ ' e $7^{\circ} 25^{\prime} 46^{\prime}$ 'S e entre as longitudes $36^{\circ} 54^{\prime} 30^{\prime \prime}$ e $36^{\circ} 54^{\prime} 35^{\prime \prime}$ W. Essa Reserva tem uma dimensão de 3.505 ha. A vegetação ribeirinha pesquisada encontra-se situada numa altitude que varia de 564 a $579 \mathrm{~m}$. Nesse local o leito do riacho apresenta cerca de $12 \mathrm{~m}$ de largura média.

Riacho dos Mares - possui cerca de $12 \mathrm{~km}$ de extensão e área de drenagem de $33 \mathrm{~km}^{2}$. Este riacho, localizado no município de São João do Cariri, está dentro dos limites da sub-bacia do riacho do Farias. A área ribeirinha pesquisada nesse curso d'água ( 3,4 ha) está inserida na propriedade Avelós (750 ha) e tem a sua localização marcada entre as latitudes $7^{\circ} 31^{\prime}$ '53" e $7^{\circ} 31^{\prime} 38^{\prime}$ " S e entre as longitudes $36^{\circ} 33^{\prime} 39^{\prime \prime}$ e $36^{\circ} 33^{\prime} 06^{\prime \prime}$ W. Neste trecho o riacho é conhecido pelos ribeirinhos como riacho Avelós, o canal é estreito com cerca de $7 \mathrm{~m}$ de largura média e a altitude varia de 536 a $550 \mathrm{~m}$.

Riacho do Farias - localizado na sub-bacia do riacho do Farias, esse riacho possui $20 \mathrm{~km}$ de extensão e bacia de drenagem de $71 \mathrm{~km}^{2}$. Neste curso d'água, a área ribeirinha estudada (3,3 ha) está definida dentro dos limites da propriedade Gangorra (450 ha), município de São João do Cariri, e tem sua localização marcada entre as latitudes $7^{\circ} 25^{\prime} 33^{\prime \prime}$ e $7^{\circ} 25^{\prime} 15^{\prime \prime}$ S e entre as longitudes $36^{\circ} 29^{\prime} 21^{\prime \prime}$, e $36^{\circ} 29^{\prime} 17^{\prime}$ 'W. Nesta área o riacho é conhecido popularmente como riacho da Gangorra, o canal se apresenta com aproximadamente $45 \mathrm{~m}$ de largura média e a altitude varia de 454 a $470 \mathrm{~m}$.

As informações sobre precipitação, temperatura, umidade relativa do ar e evaporação referentes ao período de janeiro/1996 a dezembro/2005, foram fornecidos pela Bacia Escola de São João do Cariri/Laboratório de Recursos Hídricos do Departamento de Engenharia Civil/Universidade Federal de Campina Grande. No período, a média anual de precipitação foi de $486,9 \mathrm{~mm}$. A temperatura média anual do ar variou de 23,6 a $27,4{ }^{\circ} \mathrm{C}$, sendo as menores temperaturas entre os meses de julho e agosto, ficando os meses de novembro e dezembro com as temperaturas maiores. A umidade relativa média mensal do ar atingiu o máximo de $75 \%$ em junho e julho e o mínimo ocorreu na estação seca nos meses de novembro e dezembro com $64 \%$ de umidade. A evaporação é significativamente elevada na região, uma vez que chegou a atingir até $2.697 \mathrm{~mm}$ por ano.

A vegetação ribeirinha é predominantemente arbórea, com ocorrência de espécies arbustivas bastante ramificadas a partir da base e com presença de estrato herbáceo que se mostra abundante no período chuvoso. Com exceção da vegetação do riacho do Cazuzinha, inserida em uma RPPN, observou-se que a vegetação dos riachos dos Mares e do Farias têm seu uso e ocupação marcados principalmente pelo pastoreio extensivo.

\section{Coleta e análise dos dados}

As atividades se apoiaram na análise de cartas e mapas de vegetação e excursões exploratórias realizadas inicialmente em vários pontos ao longo da bacia do Taperoá (Lacerda \& Barbosa 2006). Os ambientes mais conservados foram selecionados para o levantamento florístico da vegetação ribeirinha distribuída ao longo de três riachos intermitentes. A escolha deste tipo de levantamento se apóia na base teórica que o define como aquele que permite efetuar comparações relativamente simples e eficientes entre áreas (van den Berg \& Oliveira-Filho 2000). 
As coletas botânicas da vegetação arbustivo-arbórea foram realizadas mensalmente no período de janeiro/2004 a junho/2006 e se processaram de forma assistemática. Diante dos raros estudos existentes sobre a vegetação ribeirinha em áreas de caatinga e, portanto sem indicativos conclusivos se as dimensões das faixas de mata ciliar acompanham ou não os respectivos tamanhos apontados na legislação vigente, estabeleceu-se para este estudo a largura mínima da faixa ciliar, ou seja, $30 \mathrm{~m}$ a partir da margem do curso d'água. Além disso, a amostragem foi realizada apenas em uma das margens dos ambientes ribeirinhos estudados (margem direita para os riachos dos Mares e do Cazuzinha e margem esquerda para o riacho do Farias).

Exemplares férteis de cada espécie foram coletados, herborizados e incorporados ao Herbário Lauro Pires Xavier (JPB) da Universidade Federal da Paraíba. A identificação das espécies e/ou confirmação se processou através de consultas a especialistas e por comparação usando bibliografia especializada e análise das exsicatas depositadas no herbário JPB. As espécies foram organizadas por família no sistema APG III (Angiosperm... 2009), incluindo-se informação sobre o hábito das mesmas. A grafia da autoria das espécies e suas respectivas abreviações foram verificadas através de Brummitt \& Powell (1992). Os nomes populares estão de acordo com o conhecimento local utilizando-se como material de coleta entrevistas com os atores chaves das comunidades presentes nos trechos ribeirinhos amostrados.

Para a comparação florística das áreas estudadas com outros levantamentos de vegetação ribeirinha na bacia do Rio Taperoá, foram reunidas oito listas, incluindo as do presente estudo e realizada uma análise de agrupamento. Nesta análise foi utilizada uma matriz de presença/ausência dos táxons identificados ao nível específico e o índice de similaridade de Jaccard (Hubálek 1982, Kent \& Coker 1992) e a técnica de ligação da média de grupo - UPGMA (Chatfield \& Collins 1983) usando o programa NTSYSpc.

\section{Resultados e Discussão}

\section{Composição florística: uma abordagem geral das áreas ribeirinhas amostradas}

A vegetação arbustivo-arbórea nos três riachos amostrados foi representada por 91 espécies, ficando 81 identificadas no nível de espécie, cinco no genérico, três no nível de família e duas permaneceram indeterminadas (Tabela 1 ). As espécies identificadas ficaram distribuídas em 27 famílias e 67 gêneros. O componente predominante foi o arbóreo com 60 espécies, ficando, desse número, uma indeterminada e as restantes distribuídas por 24 famílias.

O total de espécies arbóreas e arbustivas observado (91) para as matas ribeirinhas é considerado elevado quando comparado com os números apresentados por Rodrigues \& Nave (2004) que analisaram 43 trabalhos realizados em florestas ciliares do Brasil extra-amazônico, em condições de clima e de altitude muito variáveis. Segundo esses autores o número de espécies arbustivo-arbóreas amostrado variou de 23 até 247 espécies nos trabalhos apresentados. O grande número de espécies encontrado nas áreas ribeirinhas de caatinga amostradas é ainda ressaltado quando se compara os resultados gerados com outros levantamentos nos diferentes tipos caducifólios do semiárido (Araújo et al. 1998, Ferraz et al. 1998, Rodal et al. 1998, Araújo et al. 1999, Lemos \& Rodal 2002, Pereira et al. 2002, Rodal \& Nascimento 2002, Alcoforado-Filho et al.

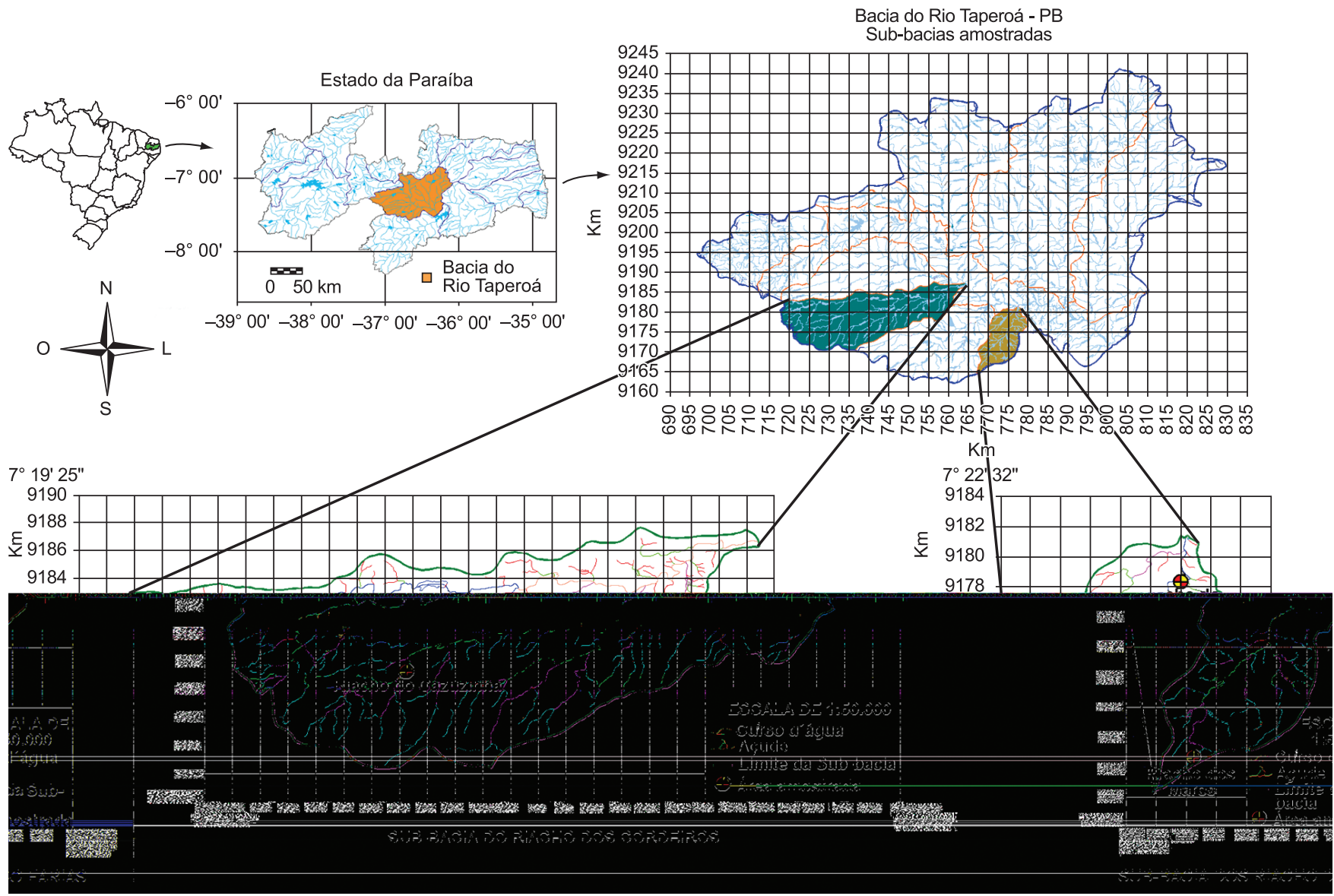

Figura 1. Localização das áreas ribeirinhas amostradas na bacia hidrográfica do Rio Taperoá, semiárido paraibano. Adaptado de Lacerda \& Barbosa (2006). Figure 1. Location of the studied areas in the basin of the Taperoá River, in the paraiban semiarid. Adapted from Lacerda \& Barbosa (2006). 
Lacerda, A.V. et al.

Tabela 1. Lista das famílias e espécies registradas no levantamento florístico realizado em três áreas ribeirinhas na bacia do rio Taperoá, semiárido paraibano, com seus respectivos nomes populares, hábitos, números de coleta e locais de ocorrência. Háb. $=$ Hábito, Arv = Árvore, Arb = Arbusto, $\mathrm{NC}=$ número de coleta de A. V. Lacerda referente ao voucher depositado no Herbário Lauro Pires Xavier (JPB), RF = Riacho do Farias, $\mathrm{RM}=\mathrm{Riacho}$ dos Mares e RC $=\mathrm{Riacho}$ do Cazuzinha.

Table 1. Families and species of shrubs and trees registered in the study the floristic composition in three riparian vegetation areas in the basin of the Taperoá river, paraiban semiarid, with your common names, Life-form, collection numbers and occurrence places. Háb. $=$ Life-form, Arv $=$ Tree, Arb $=$ Shrub, $\mathrm{NC}=$ number of A. V. Lacerda regarding the voucher deposited in the Herbarium Lauro Pires Xavier (JPB), RF $=$ Farias Stream, RM $=$ Mares Stream and $\mathrm{RC}=$ Cazuzinha Stream.

\begin{tabular}{|c|c|c|c|c|c|c|}
\hline $\begin{array}{l}\text { Família } \\
\text { Espécies }\end{array}$ & Nome Popular & Háb. & $\mathbf{R F}$ & $\mathbf{R M}$ & $\mathbf{R C}$ & NC \\
\hline \multicolumn{7}{|l|}{ 1. ANACARDIACEAE } \\
\hline 1 Myracrodruon urundeuva Allemão & Aroeira & Arv & $X$ & $\mathrm{X}$ & $\mathrm{X}$ & 240 \\
\hline 2 Schinopsis brasiliensis Engl. & Baraúna & Arv & $\mathrm{X}$ & $X$ & $\mathrm{X}$ & 218 \\
\hline 3 Spondias tuberosa Arruda & Umbuzeiro & Arv & $\mathrm{X}$ & $\mathrm{X}$ & $\mathrm{X}$ & 283 \\
\hline \multicolumn{7}{|l|}{ 2. ANNONACEAE } \\
\hline 4 Rollinia leptopetala R. E. Fr. & Pinha brava & Arb & - & - & $\mathrm{X}$ & 349 \\
\hline \multicolumn{7}{|l|}{ 3. APOCYNACEAE } \\
\hline 5 Allamanda blanchetii A. DC. & Quatro pataca & Arb & $\mathrm{X}$ & $X$ & - & 59 \\
\hline 6 Aspidosperma pyrifolium Mart. & Pereiro & Arv & $\mathrm{X}$ & $\mathrm{X}$ & $\mathrm{X}$ & 279 \\
\hline \multicolumn{7}{|l|}{ 4. BIGNONIACEAE } \\
\hline $\begin{array}{l}7 \text { Tabebuia aurea (Silva Manso) Benth. \& Hook. f. } \\
\text { ex S. Moore * }\end{array}$ & Craibeira & Arv & $\mathrm{X}$ & $\mathrm{X}$ & - & 217 \\
\hline 8 Tabebuia impetiginosa (Mart. ex DC.) Standl. & Ipê-roxo & Arv & - & - & $\mathrm{X}$ & 168 \\
\hline \multicolumn{7}{|l|}{ 5. BIXACEAE } \\
\hline 9 Cochlospermum insigne A. St.-Hil. ${ }^{*}$ & Algodão bravo & Arv & $\mathrm{X}$ & $\mathrm{X}$ & - & 511 \\
\hline \multicolumn{7}{|l|}{ 6. BORAGINACEAE } \\
\hline 10 Cordia leucocephala Moric. & Moleque duro & Arb & - & $\mathrm{X}$ & - & 467 \\
\hline 11 Cordia trichotoma (Vell.) Arráb. ex Steud. & Louro, Frei Jorge & Arv & $\mathrm{X}$ & $\mathrm{X}$ & $\mathrm{X}$ & 463 \\
\hline 12 Tournefortia rubicunda Salzm. ex DC. & Maria preta & Arb & - & $\mathrm{X}$ & - & 549 \\
\hline \multicolumn{7}{|l|}{ 7. BRASSICACEAE } \\
\hline 13 Capparis flexuosa (L.) L. & Feijão bravo & Arv & $\mathrm{X}$ & $\mathrm{X}$ & $\mathrm{X}$ & 34 \\
\hline 14 Capparis jacobinae Moric. ex Eichler & Icó & Arv & - & $\mathrm{X}$ & $\mathrm{X}$ & 266 \\
\hline \multicolumn{7}{|l|}{ 8. BURSERACEAE } \\
\hline 15 Commiphora leptophloeos (Mart.) J. B. Gillett & Amburana de cambão & Arv & $\mathrm{X}$ & $\mathrm{X}$ & $\mathrm{X}$ & 275 \\
\hline \multicolumn{7}{|l|}{ 9. CACTACEAE } \\
\hline 16 Cereus jamacaru DC. & Mandacaru, Cardeiro & Arv & $\mathrm{X}$ & $\mathrm{X}$ & $\mathrm{X}$ & 558 \\
\hline $\begin{array}{l}17 \text { Pilosocereus gounellei (F. A. C. Weber) Byles } \\
\text { \& G. D. Rowley }\end{array}$ & Xique-xique, Alastrado & Arb & $\mathrm{X}$ & $\mathrm{X}$ & $\mathrm{X}$ & 557 \\
\hline $\begin{array}{l}18 \text { Pilosocereus pachycladus subsp. pernambucensis } \\
\text { (Ritter) Zappi }\end{array}$ & Facheiro & Arv & $\mathrm{X}$ & $\mathrm{X}$ & $\mathrm{X}$ & 556 \\
\hline \multicolumn{7}{|l|}{ 10. CELASTRACEAE } \\
\hline 19 Maytenus rigida Mart. & Bonome & Arv & $\mathrm{X}$ & $\mathrm{X}$ & $\mathrm{X}$ & 235 \\
\hline \multicolumn{7}{|l|}{ 11. COMBRETACEAE } \\
\hline 20 Combretum laxum Jacq. * & Mofumbo & Arb & $\mathrm{X}$ & - & - & 483 \\
\hline 21 Combretum leprosum Mart. & Mofumbo & Arb & $\mathrm{X}$ & $\mathrm{X}$ & $\mathrm{X}$ & 82 \\
\hline 22 Combretum pisonioides Taub. & Canela de veado & Arv & $\mathrm{X}$ & $\mathrm{X}$ & $\mathrm{X}$ & 206 \\
\hline \multicolumn{7}{|l|}{ 12. ERYTHROXYLACEAE } \\
\hline 23 Erythroxylum revolutum Mart. & & Arv & $\mathrm{X}$ & $\mathrm{X}$ & $\mathrm{X}$ & 372 \\
\hline \multicolumn{7}{|l|}{ 13. EUPHORBIACEAE } \\
\hline 24 Cnidoscolus quercifolius Pohl & Favela & Arv & $\mathrm{X}$ & - & - & 24 \\
\hline 25 Croton echioides Baill. & Caatinga branca & Arb & - & $X$ & $\mathrm{X}$ & 354 \\
\hline 26 Croton blanchetianus Baill. & Marmeleiro & Arb & $\mathrm{X}$ & $\mathrm{X}$ & $\mathrm{X}$ & 39 \\
\hline 27 Croton urticifolius Lam. & Velame & Arb & - & $X$ & - & 428 \\
\hline 28 Croton adenocalyx Baill. & Velame brabo & Arb & - & - & $\mathrm{X}$ & 388 \\
\hline
\end{tabular}

* Espécies exclusivas deste levantamento. 
Tabela 1. Continuação...

\begin{tabular}{|c|c|c|c|c|c|c|}
\hline $\begin{array}{l}\text { Família } \\
\text { Espécies }\end{array}$ & Nome Popular & Háb. & RF & $\mathbf{R M}$ & $\mathbf{R C}$ & NC \\
\hline 29 Ditaxis malpighiacea(Ule) Pax \& K.Hoffm. & Pau matias & Arb & $\mathrm{X}$ & $\mathrm{X}$ & $\mathrm{X}$ & 425 \\
\hline 30 Jatropha mollissima (Pohl) Baill. & Pinhão & Arb & $\mathrm{X}$ & $\mathrm{X}$ & $\mathrm{X}$ & 369 \\
\hline 31 Manihot catingae Ule & Maniçoba & Arv & $\mathrm{X}$ & $X$ & $\mathrm{X}$ & 87 \\
\hline 32 Sapium glandulosum (L.) Morong & Burra leiteira & Arv & - & $\mathrm{X}$ & $X$ & 395 \\
\hline 33 Sebastiania macrocarpa Müll. Arg. * & Pau leite & Arv & $\mathrm{X}$ & $X$ & $\mathrm{X}$ & 169 \\
\hline 34 Euphorbia phosphorea Mart. & Avelós & Arb & - & $\mathrm{X}$ & - & 376 \\
\hline 35 Euphorbiaceae 1 & & Arv & - & - & $\mathrm{X}$ & 249 \\
\hline \multicolumn{7}{|l|}{ 14. FABACEAE } \\
\hline \multicolumn{7}{|l|}{ 14.1 FABACEAE subfam. CAESALPINIOIDEAE } \\
\hline 36 Bauhinia cheilantha (Bong.) Steud. & Mororó & Arb & $\mathrm{X}$ & $\mathrm{X}$ & $\mathrm{X}$ & 464 \\
\hline 37 Libidibia ferrea (Mart. ex Tul.) L.P. Queiroz & Pau ferro & Arv & $\mathrm{X}$ & $\mathrm{X}$ & $\mathrm{X}$ & 32 \\
\hline 38 Caesalpinia pyramidalis Tul. & Catingueira & Arv & $\mathrm{X}$ & $X$ & $\mathrm{X}$ & 36 \\
\hline 39 Hymenaea courbaril L. & Jatobá & Arv & - & $\mathrm{X}$ & - & 258 \\
\hline 40 Peltogyne pauciflora Benth. * & & Arv & $\mathrm{X}$ & - & - & 299 \\
\hline $\begin{array}{l}41 \text { Senna macranthera var. pudibunda (Benth.) H. S. } \\
\text { Irwin \& Barneby }\end{array}$ & Flor de São João & Arv & - & $\mathrm{X}$ & $\mathrm{X}$ & 102 \\
\hline 42 Senna martiana (Benth.) H. S. Irwin \& Barneby & Canafístula brava & Arb & - & $\mathrm{X}$ & - & 48 \\
\hline 43 Senna spectabilis (DC.) H. S. Irwin \& Barneby & Canafístula & Arv & - & - & $\mathrm{X}$ & 452 \\
\hline \multicolumn{7}{|l|}{ 14.2 FABACEAE subfam. FABOIDEAE } \\
\hline 44 Amburana cearensis (Allemão) A. C. Sm. & $\begin{array}{l}\text { Amburana de cheiro, } \\
\text { Cumarú }\end{array}$ & Arv & - & $\mathrm{X}$ & $\mathrm{X}$ & 152 \\
\hline 45 Erythrina velutina Willd. & Mulungu & Arv & $\mathrm{X}$ & - & $\mathrm{X}$ & 227 \\
\hline 46 Lonchocarpus sericeus (Poir.) Kunth ex DC. & Ingazeira & Arv & $\mathrm{X}$ & - & $\mathrm{X}$ & 38 \\
\hline 47 Lonchocarpus cf. obtusus Benth. * & Ingaí, Rabo de cavalo & Arv & $\mathrm{X}$ & - & $\mathrm{X}$ & 246 \\
\hline 48 Luetzelburgia auriculata (Allemão) Ducke & Pau de serrote & Arv & $X$ & $X$ & - & 506 \\
\hline 49 Myroxylon peruiferum L.f. & Bálsamo & Arv & - & - & $\mathrm{X}$ & 288 \\
\hline 50 Poecilanthe ulei (Harms) Arroyo \& Rudd * & Chorão & Arv & - & - & $\mathrm{X}$ & 254 \\
\hline 51 Fabaceae - Faboideae 1 & Crabraiba & Arv & - & - & $\mathrm{X}$ & 547 \\
\hline \multicolumn{7}{|l|}{ 14.3 FABACEAE subfam. MIMOSOIDEAE } \\
\hline 52 Acacia paniculata Willd. & Gameleira brava & Arv & - & - & $\mathrm{X}$ & 329 \\
\hline 53 Anadenanthera colubrina (Vell.) Brenan & Angico & Arv & $X$ & $X$ & $\mathrm{X}$ & 262 \\
\hline 54 Calliandra sp. 1 & Mucumbu de ema & Arb & - & $X$ & - & 555 \\
\hline 55 Chloroleucon foliolosum (Benth.) G. P. Lewis & $\begin{array}{l}\text { Jurema açu, } \\
\text { Jurema branca }\end{array}$ & Arv & $\mathrm{X}$ & $X$ & $\mathrm{X}$ & 37 \\
\hline 56 Mimosa ophthalmocentra Mart. ex Benth. & Jurema de imbira & Arv & $\mathrm{X}$ & $X$ & $\mathrm{X}$ & 99 \\
\hline 57 Mimosa paraibana Barneby & Calumbi & Arb & - & $X$ & - & 138 \\
\hline 58 Mimosa tenuiflora (Willd.) Poir. & Jurema preta & Arv & $\mathrm{X}$ & $X$ & $\mathrm{X}$ & 260 \\
\hline 59 Piptadenia stipulacea (Benth.) Ducke & Jurema branca & Arv & $X$ & $\mathrm{X}$ & $\mathrm{X}$ & 173 \\
\hline \multicolumn{7}{|l|}{ 15. MALVACEAE } \\
\hline 60 Ceiba glaziovii (Kuntze) K. Schum. & Barriguda & Arv & - & - & $\mathrm{X}$ & 512 \\
\hline $\begin{array}{l}61 \text { Pseudobombax marginatum (A. St.-Hil., Juss. \& } \\
\text { Cambess.) A. Robyns }\end{array}$ & Embiratanha & Arv & $\mathrm{X}$ & $X$ & $\mathrm{X}$ & 286 \\
\hline 62 Helicteres brevispira A. St.-Hil. & Guaxumbu & Arb & - & - & $\mathrm{X}$ & 198 \\
\hline 63 Melochia pyramidata L. & Capa bode & Arb & $X$ & $X$ & - & 446 \\
\hline \multicolumn{7}{|l|}{ 16. MYRTACEAE } \\
\hline 64 Eugenia uvalha Cambess. & Ubaia & Arv & $\mathrm{X}$ & - & $\mathrm{X}$ & 540 \\
\hline 65 Myrtaceae 1 & & Arv & - & - & $\mathrm{X}$ & 328 \\
\hline \multicolumn{7}{|l|}{ 17. NYCTAGINACEAE } \\
\hline 66 Guapira laxa (Netto) Furlan & João mole, Piranha & Arv & $X$ & $\mathrm{X}$ & $\mathrm{X}$ & 256 \\
\hline
\end{tabular}

* Espécies exclusivas deste levantamento. 
Tabela 1. Continuação...

\begin{tabular}{|c|c|c|c|c|c|c|}
\hline $\begin{array}{l}\text { Família } \\
\text { Espécies }\end{array}$ & Nome Popular & Háb. & RF & $\mathbf{R M}$ & RC & NC \\
\hline \multicolumn{7}{|l|}{ 18. OLACACEAE } \\
\hline 67 Ximenia americana L. & Ameixa brava & Arv & $\mathrm{X}$ & $\mathrm{X}$ & - & 532 \\
\hline \multicolumn{7}{|l|}{ 19. POLYGONACEAE } \\
\hline 68 Triplaris gardneriana Wedd. & Cauaçu & Arv & $\mathrm{X}$ & - & $X$ & 174 \\
\hline \multicolumn{7}{|l|}{ 20. RHAMNACEAE } \\
\hline 69 Rhamnidium molle Reissek & Sassafrás & Arv & $\mathrm{X}$ & - & $\mathrm{X}$ & 213 \\
\hline 70 Ziziphus cotinifolia Reissek & Juazeiro & Arv & $\mathrm{X}$ & - & - & 281 \\
\hline 71 Ziziphus joazeiro Mart. & Juazeiro & Arv & $\mathrm{X}$ & $\mathrm{X}$ & $\mathrm{X}$ & 73 \\
\hline \multicolumn{7}{|l|}{ 21. RUBIACEAE } \\
\hline 72 Alibertia sp. 1 & & Arb & $\mathrm{X}$ & $\mathrm{X}$ & - & 304 \\
\hline 73 Coutarea hexandra (Jacq.) K. Schum. & & Arv & - & - & $\mathrm{X}$ & 348 \\
\hline 74 Guettarda angelica Mart. ex Müll. Arg. & & Arb & - & $\mathrm{X}$ & $\mathrm{X}$ & 364 \\
\hline 75 Randia formosa (Jacq.) K. Schum. ${ }^{*}$ & Rosário de nego & Arv & - & - & $\mathrm{X}$ & 360 \\
\hline 76 Tocoyena formosa (Cham. \& Schltdl.) K. Schum. & Genipapo & Arv & $\mathrm{X}$ & $\mathrm{X}$ & $\mathrm{X}$ & 375 \\
\hline 77 Tocoyena sellowiana (Cham. \& Schltdl.) K. Schum. * & Genipapo & Arv & $\mathrm{X}$ & $\mathrm{X}$ & $\mathrm{X}$ & 46 \\
\hline \multicolumn{7}{|l|}{ 22. SALICACEAE } \\
\hline 78 Prockia crucis P. Browne ex L. & & Arb & - & - & $\mathrm{X}$ & 351 \\
\hline \multicolumn{7}{|l|}{ 23. SAPINDACEAE } \\
\hline 79 Allophylus quercifolius (Mart.) Radlk. & Batinga & Arv & $\mathrm{X}$ & $\mathrm{X}$ & $\mathrm{X}$ & 371 \\
\hline \multicolumn{7}{|l|}{ 24. SAPOTACEAE } \\
\hline $\begin{array}{l}80 \text { Sideroxylon obtusifolium (Roem. \& Schult.) T. D. } \\
\text { Penn. }\end{array}$ & Quixabeira & Arv & $\mathrm{X}$ & $\mathrm{X}$ & $\mathrm{X}$ & 151 \\
\hline \multicolumn{7}{|l|}{ 25. SOLANACEAE } \\
\hline 81 Capsicum parvifolium Sendtn. & & Arb & $\mathrm{X}$ & $X$ & $X$ & 353 \\
\hline 82 Nicotiana glauca Graham & Oliveira & Arb & $\mathrm{X}$ & - & $\mathrm{X}$ & 521 \\
\hline 83 Solanum rhytidoandrum Sendtn. & Jurubeba & Arb & - & - & $\mathrm{X}$ & 176 \\
\hline \multicolumn{7}{|l|}{ 26. VERBENACEAE } \\
\hline 84 Lantana camara L. & Chumbinho & Arb & - & $X$ & $\mathrm{X}$ & 473 \\
\hline 85 Lippia gracilis Schauer & Alecrim & Arb & $\mathrm{X}$ & $X$ & & 157 \\
\hline 86 Lippia sp. 1 & Camará de espeto & Arb & - & $X$ & $\mathrm{X}$ & 553 \\
\hline 87 Vitex gardneriana Schauer * & Jatiúca & Arb & $\mathrm{X}$ & $X$ & - & 97 \\
\hline 88 Vitex sp. 1 & & Arv & - & $\mathrm{X}$ & - & 496 \\
\hline \multicolumn{7}{|l|}{ 27. VOCHYSIACEAE } \\
\hline 89 Callisthene sp. 1 & Vinhaca & Arv & - & $X$ & - & 366 \\
\hline \multicolumn{7}{|l|}{ INDETERMINADAS } \\
\hline 90 Indeterminada 1 & & Arv & $X$ & $\mathrm{X}$ & $X$ & 485 \\
\hline 91 Indeterminada 2 & & Arb & - & - & $\mathrm{X}$ & 549 \\
\hline
\end{tabular}

* Espécies exclusivas deste levantamento.

2003, Cavalcanti et al. 2003, Lemos 2004, Santana 2005). Nestes 11 trabalhos, as espécies arbóreas e arbustivas registradas variaram de 22 a 154 espécies. Além disso, o total de espécies encontradas é também um número elevado quando comparado com o total de espécies lenhosas (475) levantadas para todas as ecorregiões da caatinga (Sampaio \& Gamarra-Rojas 2003).

A significativa riqueza da vegetação ciliar da região semiárida é também observada no trabalho de Miranda \& Silva (1989). Outros autores como Rezende (1998) discutem que a vegetação presente ao longo dos corpos d'água apresenta um elevado número de espécies, sendo este superior ao encontrado em outras formações florestais. Rodrigues \& Nave (2004) destacam que este fato é condicionado pela natureza ecotonal da faixa ciliar, que é ocupada por mais de um tipo vegetacional ou mesmo por formações fitofisionômicas distintas, que diferem entre si em termos de composição florística. OliveiraFilho et al. (1990) apontam também que uma riqueza de espécies relativamente elevada é característica comum em florestas ciliares devido a uma heterogeneidade ambiental comumente superior à de florestas de terra firme próximas. As matas presentes ao longo dos cursos d'água se constituem de comunidades vegetais caracterizadas pela combinação diferenciada da atuação dos fatores bióticos e abióticos, que resultam em manchas com florística e/ou estrutura própria dessa vegetação.

Observando a totalidade das áreas estudadas, as famílias com maior número de espécies e gêneros no estrato arbustivo-arbóreo foram Fabaceae com 24 espécies (26,4\%) e 17 gêneros (25,4\%), Euphorbiaceae representada com 12 espécies $(13,2 \%)$ e oito gêneros $(11,9 \%)$ e Rubiaceae apresentando seis espécies $(6,6 \%)$ e 
cinco gêneros (7,5\%). Essas três famílias possuem juntas $46,2 \%$ das espécies e $44,8 \%$ dos gêneros, enquanto que as outras 24 famílias dividiram os $53,8 \%$ das espécies e 55,2\% dos gêneros restantes.

Ao avaliar 43 trabalhos realizados em florestas ciliares do Brasil extra-amazônico, Rodrigues \& Nave (2004) registraram que entre as oito famílias mais ricas citadas estão Fabaceae, Euphorbiaceae e Rubiaceae.

Em levantamentos florísticos e fitossociológicos realizados em diferentes tipos caducifólios do semiárido nordestino, Fabaceae e Euphorbiaceae foram citadas entre as famílias de maior riqueza em todos esses estudos (Araújo et al. 1998, Ferraz et al. 1998, Lemos \& Rodal 2002, Pereira et al. 2002, Rodal \& Nascimento 2002, AlcoforadoFilho et al. 2003, Cavalcanti et al. 2003, Lemos 2004, Santana 2005, Barbosa et al. 2007). Esses dados só vêm a confirmar a ampla distribuição dessas famílias nos vários ecossistemas do semiárido.

Dos 10 trabalhos em áreas de caatinga relacionados acima, quatro citaram Rubiaceae (Ferraz et al. 1998, Pereira et al. 2002, Rodal \& Nascimento 2002, Alcoforado-Filho et al. 2003) entre as famílias de maior número de espécies. Particularmente no componente lenhoso da Vegetação Caducifólia Espinhosa (VCE), a ocorrência de Rubiaceae tem sido registrada apenas em áreas mais úmidas como São José do Belmonte e Caruaru, ambas no Estado de Pernambuco (Tavares et al. 1969, Alcoforado-Filho et al. 2003), Barbalha no Estado do Ceará (Tavares et al. 1974) e Souza na Paraíba (Gadelha-Neto \& Barbosa 2000). Autores como Ferraz et al. (1998) também destacam Rubiaceae como uma família típica de matas mais úmidas.
Relacionado à ocorrência de famílias como Myrtaceae e Bignoniaceae estas são pouco citadas para áreas de vegetação caducifólia espinhosa (caatinga) instaladas no cristalino (Lemos \& Rodal 2002). Myrtaceae também foi citada por Rodal \& Nascimento (2002) como uma família comum em ambientes mais úmidos.

A maior parte dos gêneros (53) possui apenas uma espécie, ficando 14 gêneros com mais de uma, ou seja, Croton com quatro espécies, Combretum, Mimosa e Senna com três cada e ainda Caesalpinia, Capparis, Cordia, Lippia, Lonchocarpus, Pilosocereus, Tabebuia, Tocoyena, Vitex e Ziziphus com duas. Estes dados apontam para uma tendência na vegetação ribeirinha estudada, a exemplo do que ocorre na caatinga (Araújo et al. 1995), em apresentar baixa diversidade dentro dos táxons.

Analisando as espécies encontradas nas áreas amostradas notase que do total de 91 espécies, 15 tiveram sua ocorrência registrada também para matas ciliares de São Paulo, Distrito Federal, Mato Grosso, Minas Gerais e Paraná (Tabela 2). Dentre estas, apenas Anadenanthera colubrina e Hymenaea courbaril foram as espécies mais citadas no total dos trabalhos pesquisados, aparecendo registradas cada uma para matas ciliares de quatro estados. Cordia trichotoma, Coutarea hexandra, Myroxylon peruiferum e Tabebuia impetiginosa foram registradas cada uma em matas ciliares de três estados. Esses dados refletem a ampla tolerância dessas espécies a variações ambientais. Comparando as espécies das áreas ribeirinhas de caatinga amostradas nesse trabalho com os 15 estudos apresentados observa-se que Minas Gerais foi o estado que registrou o maior número de espécies em comum (13).

Tabela 2. Lista de espécies registradas neste estudo e referenciadas em levantamentos efetuados em matas ciliares de vários Estados brasileiro e no trabalho de classificação de espécies endêmicas da caatinga.

Table 2. List of species registered in this study and cited in references in the riparian vegetation of several States Brazilian and in the study of classification of endemic species of the caatinga.

\begin{tabular}{|c|c|c|}
\hline Referências & Local/Estudo & Espécies \\
\hline $\begin{array}{l}\text { Rozza \& Ribeiro (1992), Soares-Silva } \\
\text { et al. (1992), Durigan \& Leitão Filho } \\
\text { (1995), Oliveira-Filho et al. (1995), } \\
\text { Silva et al. (1995), Pinto et al. (1997), } \\
\text { Silva Júnior et al. (1998), Mariano et al. } \\
\text { (1998), Felfili et al. (2000), van den } \\
\text { Berg \& Oliveira-Filho (2000), Botelho } \\
\text { \& Davide (2002), Souza et al. (2003), } \\
\text { Veiga et al. (2003), Vieira et al. (2003), } \\
\text { Meyer et al. (2004) }\end{array}$ & $\begin{array}{l}\text { Matas ciliares nos estados de } \\
\text { São Paulo, Distrito Federal, } \\
\text { Mato Grosso, Minas Gerais e } \\
\text { Paraná }\end{array}$ & $\begin{array}{c}\text { Acacia paniculata, Amburana cearensis, Anadenanthera } \\
\text { colubrina, Libidibia ferrea, Cordia trichotoma, Coutarea } \\
\text { hexandra, Hymenaea courbaril, Lantana camara, } \\
\text { Myracrodruon urundeuva, Myroxylon peruiferum, Prockia } \\
\text { crucis, Senna macranthera var. pudibunda, Senna spectabilis, } \\
\text { Tabebuia impetiginosa e Triplaris gardneriana. }\end{array}$ \\
\hline Miranda \& Silva (1989 & $\begin{array}{l}\text { Matas ciliares das depressões } \\
\text { inundáveis e eixos } \\
\text { hidrográficos do semiárido } \\
\text { pernambucano }\end{array}$ & $\begin{array}{c}\text { Amburana cearensis, Anadenanthera colubrina, Aspidosperma } \\
\text { pyrifolium, Bauhinia cheilantha, Libidibia ferrea, Caesalpinia } \\
\text { pyramidalis, Capparis flexuosa, Cereus jamacaru, Cnidoscolus } \\
\text { phylacanthus, Combretum pisonioides, Commiphora } \\
\text { leptophloeos, Cordia leucocephala, Erythrina velutina, } \\
\text { Lantana camara, Mimosa tenuiflora, Myracrodruon urundeuva, } \\
\text { Pilosocereus gounellei, Schinopsis brasiliensis, Spondias } \\
\text { tuberosa, Tocoyena formosa e Ziziphus joazeiro }\end{array}$ \\
\hline Giulietti et al. 2002 & $\begin{array}{c}\text { Espécies endêmicas da } \\
\text { caatinga }\end{array}$ & $\begin{array}{c}\text { Allamanda blanchetii, Aspidosperma pyrifolium, Caesalpinia } \\
\text { pyramidalis, Capparis flexuosa, Capparis jacobinae, } \\
\text { Ceiba glaziovii, Cereus jamacaru, Combretum pisonioides, } \\
\text { Commiphora leptophloeos, Cordia leucocephala, Guettarda } \\
\text { angelica, Helicteres brevispira, Jatropha mollissima, Lippia } \\
\text { gracilis, Manihot catingae, Maytenus rigida, Mimosa } \\
\text { ophthalmocentra, Pilosocereus gounellei, Pilosocereus } \\
\text { pachycladus subsp. pernambucensis, Pseudobombax } \\
\text { marginatum, Rhamnidium molle, Rollinia leptopetala, Senna } \\
\text { martiana, Spondias tuberosa, Ziziphus cotinifolia e Ziziphus } \\
\text { joazeiro }\end{array}$ \\
\hline
\end{tabular}


Considerando ainda a listagem de espécies apresentadas neste trabalho, percebe-se que algumas estão citadas também no levantamento realizado por Miranda \& Silva (1989) nas matas ciliares das depressões inundáveis e eixos hidrográficos do semiárido pernambucano (Tabela 2).

Mostrando também a seletividade de algumas espécies em áreas de caatinga por ambientes mais úmidos, Rodal \& Nascimento (2002) referenciam Allophylus quercifolius, Libidibia ferrea, Sideroxylon obtusifolium, Tabebuia impetiginosa e Ziziphus joazeiro como espécies de ocorrência em áreas mais úmidas da Vegetação Caducifólia Espinhosa (VCE) nordestina. Pereira et al. (2002) também discutem que além de Tabebuia impetiginosa, Coutarea hexandra e Eugenia uvalha são pouco frequentes nos levantamentos da vegetação caducifólia espinhosa, sendo raramente encontradas em áreas de caatinga submetidas a maior semiaridez. Especialmente relacionado à Eugenia uvalha, AlcoforadoFilho et al. (2003) destacam que a presença freqüente dessa espécie é incomum em áreas de VCE. Portanto, os dados apontam para uma tendência de seletividade dessas espécies por locais mais úmidos a exemplo daqueles encontrados ao longo de rios e riachos intermitentes em áreas de caatinga no semiárido.

Observando a questão do endemismo, das 91 espécies encontradas 26 são endêmicas da caatinga (Giulietti et al. 2002) conforme Tabela 2.

A exemplo de trabalhos que investigaram as ligações florísticas das matas ciliares com outros ambientes (Rodrigues et al. 2003), observase que uma parcela significativa das espécies ribeirinhas listadas nesse trabalho são compartilhadas com formações vegetacionais típicas de caatinga. Colaborando com essa discussão, Rodrigues (1989) coloca que como a atuação diferenciada dos fatores abióticos ocorre no espaço e no tempo, esses fatores acabam por selecionar espécies adaptadas à interferência da água nas áreas, como freqüência e intensidade de alagamentos, baixa profundidade do lençol freático, alta concentração de matéria orgânica, etc., e espécies típicas das formações adjacentes àquelas ciliares, cuja ocorrência não está relacionada com a presença do curso d'água, como áreas de barranco e locais com lençol freático mais profundo. Segundo este autor, o gradiente de interação entre a formação florestal ciliar propriamente dita e a formação adjacente se torna ainda mais complexo com a crescente quantidade de sedimentos sólidos que são depositados nas margens desses cursos d'água, em função dos processos de assoreamento, promovendo a criação de novos nichos que sofrerão processos de sucessão e seleção das espécies para ocupação, em função das características abióticas atuantes naquele ponto. Portanto, considerando a disponibilidade de água como um dos principais fatores condicionantes da heterogeneidade da composição da vegetação ciliar observa-se que no semiárido a ligação florística entre matas ribeirinhas e a vegetação de caatinga se mostra evidenciada uma vez que a intermitência das águas é uma característica marcante na semiaridez.

Para verificar a ocorrência de espécies exclusivas neste levantamento foram analisadas as listas florísticas de 12 trabalhos realizados em diferentes localidades e tipos caducifólios do semiárido (Moura \& Barbosa 1995, Araújo et al. 1998, Ferraz et al. 1998, Rodal et al. 1998, Araújo et al. 1999, Lemos \& Rodal 2002, Pereira et al. 2002, Rodal \& Nascimento 2002, Alcoforado-Filho et al. 2003, Lemos 2004, Santana 2005, Barbosa et al. 2007). Foi consultada também a relação de espécies endêmicas da caatinga (Giulietti et al. 2002). Observou-se que em nenhum levantamento acima citado ocorreram: Cochlospermum insigne, Combretum laxum, Lonchocarpus obtusus, Peltogyne pauciflora, Poecilanthe ulei, Randia formosa, Sebastiania macrocarpa, Tabebuia aurea, Tocoyena sellowiana e Vitex gardneriana. Além dessas 10 espécies, também não ocorreram nos trabalhos citados Myroxylon peruiferum e Triplaris gardneriana. A primeira citada em levantamentos de matas ciliares em Minas Gerais (Oliveira-Filho et al. 1995), Paraná (Soares-Silva et al. 1992) e São Paulo (Durigan \& Leitão Filho 1995) e a segunda referenciada por Vieira et al. (2003) como presente na vegetação ciliar em Minas Gerais.

Portanto, além das espécies compartilhadas com levantamentos de matas ciliares de outras regiões do Brasil, as espécies exclusivas registradas nesse trabalho podem também oferecer indicativos da seletividade das mesmas por áreas de matas ribeirinhas na caatinga e se configurarem como carcterísticas desses ambientes ainda pouco estudados em suas peculiaridades florísticas.

\section{Composição florística: uma abordagem por área}

Avaliando a distribuição das famílias nas três áreas ribeirinhas tem-se que os riachos do Farias e do Cazuzinha apresentaram 24 famílias cada, ficando o riacho dos Mares com 23. Para cada área, as famílias com maior número de espécies foram: riacho do Farias - Fabaceae (13), Euphorbiaceae (seis); riacho dos Mares Fabaceae (15), Euphorbiaceae (nove), Rubiaceae e Verbenaceae (quatro cada); riacho do Cazuzinha - Fabaceae (18), Euphorbiaceae (nove) e Rubiaceae (cinco). Em relação às famílias exclusivas em cada área observou-se que apenas três foram exclusivas a uma localidade: Annonaceae e Salicaceae presentes apenas no riacho do Cazuzinha e Vochysiaceae registrada na vegetação ribeirinha do riacho dos Mares. Estiveram presentes em todas as áreas 20 famílias (74,1\%).

Relacionado à distribuição das espécies, do total das 91 registradas, 56 espécies ocorreram no riacho do Farias (cinco exclusivas), 62 estiveram presentes no riacho dos Mares (10 exclusivas) e 68 levantadas no riacho do Cazuzinha (17 exclusivas). Cerca de $40 \%$ das espécies ocorreram nas três áreas (Tabela 1).

\section{Similaridade florística}

A análise de agrupamento, realizada com base em uma matriz de presença/ausência de 81 espécies identificadas nas três áreas de estudo e em outros cinco levantamentos desenvolvidos nas matas ribeirinhas da bacia do Rio Taperoá (Lacerda et al. 2004, Lacerda \& Barbosa 2006) indica diferentes níveis de similaridade entre os ambientes analisados (Figura 2).

Observou-se que os maiores valores de similaridade são compartilhados pelos riachos Farias (RF), Salgado (RS), Mares (RM) e Cazuzinha (RC). Destas quatro áreas, os riachos do Farias (RF) e Salgado (RS) foram os mais similares (64\%). A menor semelhança foi definida entre o Rio Taperoá e as demais áreas com 23\% de similaridade. Particularmente relacionado ao agrupamento das quatro áreas com maior similaridade, constituído ao nível de 52\%, observa-se que três se referem aos ambientes ribeirinhos deste estudo.

De modo geral, a análise de similaridade florística entre as áreas evidenciou uma maior semelhança entre localidades próximas. Desta forma, a proximidade geográfica entre os riachos Farias, Salgado, Mares e Cazuzinha explica a maior similaridade encontrada entre esses ambientes, o que está de acordo com o apontado por Rodrigues \& Nave (2004) ao estudarem a similaridade florística em florestas ciliares do Brasil. Para estes autores, os agrupamentos das áreas foram definidos, entre outros aspectos, em função da proximidade espacial entre as áreas. Contudo, percebe-se nesse estudo que os maiores e menores níveis de similaridade entre áreas também se explicam pelo seu histórico de uso e ocupação humana. Desta forma, com exceção da vegetação do riacho do Cazuzinha, inserida em uma RPPN, observa-se que as matas dos riachos dos Mares, Farias e Salgado têm seu uso e ocupação marcados principalmente pelo pastoreio extensivo. Por outro lado, sem considerar o Riacho Santo Antônio, que também tem a mesma atividade citada para os três últimos riachos, observa-se que as demais áreas foram submetidas a impacto antrópico muito semelhante devido à realização de atividades agropecuárias. Portanto, é perceptível que a distância geográfica aliada às peculiaridades do uso e ocupação da terra, caracterizam o conjunto de fatores responsáveis pela similaridade entre as áreas de vegetação ribeirinha pesquisadas. 


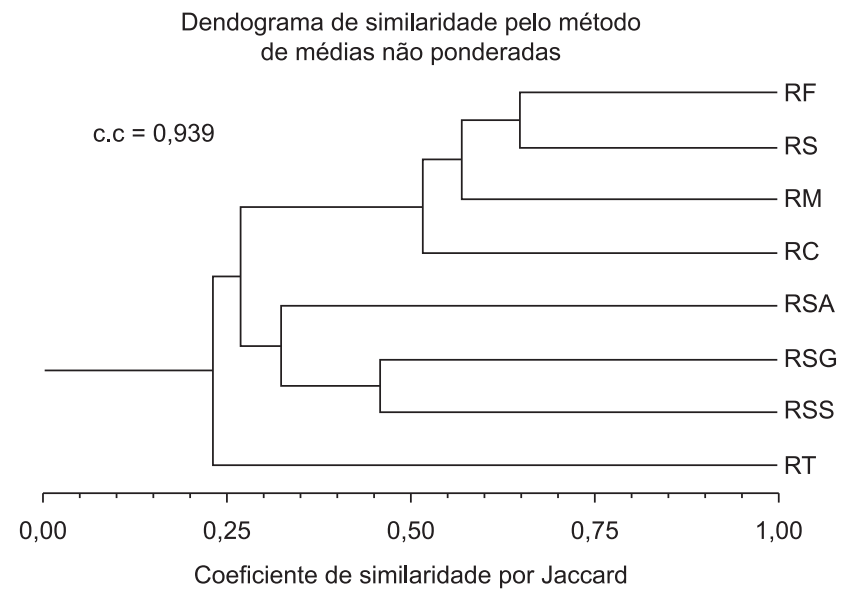

Figura 2. Similaridade florística entre as três áreas estudadas (RF - Riacho do Farias; RM - Riacho dos Mares; RC - Riacho do Cazuzinha) e outros cinco levantamentos desenvolvidos nas áreas ribeirinhas da bacia do rio Taperoá (RS - Riacho Salgado; RSA - Riacho Santo Antônio; RSG - Rio Soledade/ município de Gurjão; RSS - Rio Soledade/município de Soledade; RT - Rio Taperoá - Lacerda et al. 2004, Lacerda \& Barbosa 2006).

Figure 2. Floristic similarities between the three sample areas (RF - Stream of Farias; RM - Stream of the Mares; RC - Stream of Cazuzinha) and other five studied developed in the riverine areas of the basin of the river Taperoá (RS Stream Salgado; RSA - Stream Santo Antônio; RSG - River Soledade/municipal district of Gurjão; RSS - River Soledade/municipal district of Soledade; RT River Taperoá - Lacerda et al. 2004, Lacerda \& Barbosa 2006).

\section{Conclusão}

Considerando os resultados obtidos conclui-se que o total de espécies listado nesse trabalho é expressivo quando comparado com levantamentos realizados nos diferentes tipos de vegetação caducifólia do semiárido. Assim como em alguns levantamentos realizados na caatinga s.str., as famílias Fabaceae e Euphorbiaceae foram bem representadas em número de espécies, o que demonstra a ampla distribuição das mesmas nos vários ecossistemas do semiárido. Entretanto, a presença de famílias como Myrtaceae e Bignoniaceae corroboram outros estudos que indicam a ocorrência destas em áreas mais úmidas. Verificou-se ainda que, a exemplo do que ocorre em outras formações ciliares do país, as árreas ribeirinhas amostradas, apresentaram uma composição florística na qual a maioria das espécies são compartilhadas com as áreas adjacentes de caatinga. Um número menor, porém, é composto por espécies típicas de formações ciliares do Brasil e de espécies exclusivas aos ecossistemas ribeirinhos na Caatinga.

\section{Agradecimentos}

A todos os proprietários das áreas pesquisadas (Maria Eunice Braz, Agrício de Souza Barros Filho e José Carlos Oliveira) pela permissão para a realização do presente estudo. Aos taxonomistas do Jardim Botânico do Rio de Janeiro, Instituto de Botânica de São Paulo, UFRN, UFRPE, UEFS e UFPB, pelo auxílio na identificação do material botânico. Ao CNPq e a CAPES, pela concessão das bolsas de doutorado concedidas às primeira e segunda autoras, respectivamente, e ainda ao $\mathrm{CNPq}$ pelo auxílio financeiro e suporte técnico através do Programa de Pesquisas Ecológicas de Longa Duração (PELD) Sítio Caatinga, executado pela Universidade Federal da Paraíba, fundamental para a realização de parte dos trabalhos de campo.

\section{Referências Bibliográficas}

ALCOFORADO-FILHO, F.G., SAMPAIO, E.V.S.B. \& RODAL, M.J.N. 2003. Florística e fitossociologia de um remanescente de vegetação caducifólia espinhosa arbórea em Caruaru, Pernambuco. Acta Bot. Bras. 17(2):287-303.

ANGIOSPERM PHYLOGENY GROUP - APG III. 2009. An update of the Angiosperm Phylogeny Group classification for the orders and families of flowering plants: APG III. Bot. J. Linn. Soc. 161:105-121.

ARAÚJO, E.L., SAMPAIO, E.V.S.B. \& RODAL, M.J.N. 1995. Composição florística e fitossociologia de três áreas de caatinga de Pernambuco. Rev. Brasil. Biol. 55(4):595-607.

ARAÚJO, F.S., MARTINS, F.R. \& SHEPHERD, G.J. 1999. Variações estruturais e florísticas do carrasco no planalto da Ibiapaba, Estado do Ceará. Rev. Brasil. Biol. 59(4):663-678.

ARAÚJO, F.S., SAMPAIO, E.V.S.B., FIGUEIREDO, M.A., RODAL, M.J.N. \& FERNANDES, A.G. 1998. Composição florística da vegetação de carrasco, Novo Oriente, CE. Brasil. Rev. Brasil. Bot. 21(2):15-26.

BARBOSA, M.R.V., LIMA, I.B., LIMA J.R., CUNHA, J.P., AGRA, M.F. \& THOMAS, W.W. 2007. Vegetação e flora no cariri paraibano. Oecol. Bras. 11(3):313-322.

BOTELHO, S.A. \& DAVIDE, A.C. 2002. Métodos silviculturais para recuperação de nascentes e recomposição de matas ciliares. In Simpósio Nacional sobre Recuperação de Áreas Degradadas. Belo Horizonte, p.123-145.

BRUMMITT, R.F. \& POWELL, C.E. 1992. Authors of plant names. Royal Botanic Gardens/Kew, London.

CAVALCANTI, A.D.C., RODAL, M.J.N., SILVA, A.C.B.L., COSTA, K.C.C. \& PESSOA, L.M. 2003. Análise da distribuição espacial da vegetação em uma área prioritária para a conservação da biodiversidade da Caatinga - Betânia/Floresta, Pernambuco. In. Congresso de Ecologia do Brasil. Ceará, p.319-320.

CHATFIELD, C. \& COLLINS, A.J. 1983. Introduction to multivariate analysis. Chapman and Hall, London.

DURIGAN, G. \& LEITÃO FILHO, H.F. 1995. Florística e fitossociologia de matas ciliares do Oeste Paulista. Rev. Inst. Flor. 7(2):197-239.

FELFILI, J.M., RIBEIRO, J.F., FAGG, C.W. \& MACHADO, J.W.B. 2000. Recuperação de matas de galeria. EMBRAPA Cerrados, Planaltina.

FERRAZ, E.M.N., RODAL, M.J.N., SAMPAIO, E.V.S.B., PEREIRA, R.C.A. 1998. Composição florística em trechos de caatinga e brejo de altitude na região do Vale do Pajeú, Pernambuco. Rev. Brasil. Bot. 21(1):7-15.

GADELHA NETO, P.C. \& BARBOSA, M.R.V. 2000. Levantamento florístico e fitossociológico em um remanescente de caatinga no município de Sousa, Paraíba. Iniciados/UFPB, 5:64-87.

GIULIETTI, A.M., HARLEY, R.M., QUEIROZ, L.P., BARBOSA, M.R.V., BOCAGE NETA, A.L. \& FIGUEIREDO, M.A. 2002. Espécies endêmicas da caatinga. In Vegetação e flora da caatinga (E.V.S.B. Sampaio, A.M Giulietti, J. Virgínio \& C.F.L. Gamarra Rojas, ed.). Associação Plantas do Nordeste - APNE, Centro Nordestino de Informações sobre Plantas - CNIP, Recife, p.103-118.

HUBÁLEK, Z. 1982. Coefficients of association and similarity, based on binary (presence-abscence) data: an evaluation. Biol. Rev. Camb. Phil. Soc. 57:669-689.

KENT, M. \& COKER, P. 1992. Vegetation description and analysis: a pratical approach. John Willey \& Sons, London.

LACERDA, A.V. \& BARBOSA, F.M. 2006. Matas ciliares no domínio das caatingas. Editora Universitária/UFPB, João Pessoa.

LACERDA, A.V. 2003. A semi-aridez e a gestão em bacias hidrográficas: visões e trilhas de um divisor de idéias. Editora Universitária/UFPB, João Pessoa.

LACERDA, A.V., NORDI, N., BARBOSA, F.M. \& WATANABE, T. 2004. Estudo florístico de matas ciliares nos ambientes intermitentes da bacia do rio Taperoá, semi-árido paraibano. In Simpósio de Ecossistemas Brasileiros (S. Watanabe, coord). ACIESP, São Paulo, p.450-454. 
LEMOS, J.R. \& RODAL, M.J.N. 2002. Fitossociologia do componente lenhoso de um trecho da vegetação de caatinga no Parque Nacional Serra da Capivara, Piauí, Brasil. Acta Bot. Bras. 16(1):23-42.

LEMOS, J.R. 2004. Composição florística do Parque Nacional Serra da Capivara, Piauí, Brasil. Rodriguesia 55(85):55-66.

MARIANO, G., CRESTANA, C.S.M., GIANNOTTI, E., COUTO, H.T.Z. 1998. Regeneração natural em área à margem de represa, no município de Piracicaba, SP. Rev. Inst. Flor. 10(1):81-93

MARINHO-FILHO, J. \& GASTAL, M.L. 2004. Mamíferos das matas ciliares dos cerrados do Brasil Central. In Matas ciliares: conservação e recuperação (R.R. Rodrigues \& H.F. Leitão Filho, ed.). EDUSP/FAPESP, São Paulo. p.209-221.

MEYER, S.T., SILVA, A.F., MARCO JÚNIOR, P. \& MEIRA NETO, J.A.A. 2004. Composição florística da vegetação arbórea de um trecho de floresta de galeria do Parque Estadual do Rola-Moça na Região Metropolitana de Belo Horizonte, MG, Brasil. Acta Bot. Bras. 18(4):701-709.

MIRANDA, E.E. \& SILVA, G.C. 1989. Ecologia da vegetação de matas ciliares nas depressões inundáveis do semi-árido brasileiro. In Simpósio sobre Mata Ciliar. Fundação Cargill, São Paulo, p.192-212.

MOURA, A.C.A. \& BARBOSA, M.R.V. 1995. Lista de espécies da família Leguminosae na caatinga paraibana. Rev. Nord. Biol. 10(1):23-37.

MUELLER, C.C. 1998. Gestão de matas ciliares. In Gestão ambiental no Brasil: experiência e sucesso (I.V. Lopes, org.). Editora Fundação Getúlio Vargas, Rio de Janeiro, p.185-214.

OLIVEIRA-FILHO, A.T. 1994. Estudos ecológicos da vegetação como subsídios para programas de revegetação com espécies nativas: uma proposta metodológica. Cerne 1(1):64-72.

OLIVEIRA-FILHO, A.T., RATTER, J.A. \& SHEPHERD, G.J. 1990. Floristic composition and community structure of a central Brazilian gallery forest. Flora 184:103-117.

OLIVEIRA-FILHO, A.T., VILELA, E.A., GAVILANES, M.L. \& CARVALHO, D.A. 1995. Estudos florísticos e fitossociológicos em remanescentes de matas ciliares do Alto e Médio Rio Grande. CEMIG, Belo Horizonte.

PARAÍBA (Estado). Superintendência de Administração do Meio Ambiente - SUDEMA. 1992. Paraíba 92: perfil ambiental e estratégia. João Pessoa.

PARAÍBA (Estado). Superintendência de Administração do Meio Ambiente - SUDEMA. 2005. Zoneamento ecológico-econômico: microrregião do Cariri Ocidental da Paraíba - vulnerabilidade ambiental. João Pessoa.

PARAÍBA. 1997. Avaliação da infra-estrutura hídrica e do suporte para o sistema de gerenciamento de recursos hídricos do Estado da Paraíba. João Pessoa.

PEREIRA, I.M., ANDRADE, L.A., BARBOSA, M.R.V. \& SAMPAIO, E.V.S.B. 2002. Composição florística e análise fitossociológica do componente arbustivo-arbóreo de um remanescente florestal no agreste paraibano. Acta Bot. Bras. 16(3):357-369.

PINTO, J.R.R., RIBEIRO, G.L.S., BENVENUTTI, D. \& MARCIEL, A.A.A. 1997. Composição florística e estrutura da comunidade arbórea-arbustiva de um trecho da floresta de galeria da queda d'água Véu-de-Noiva, Parque Nacional da Chapada dos Guimarães, MT. In Contribuição ao Conhecimento Ecológico do Cerrado (L.L. Leite \& C.H. Saito, org.). Universidade de Brasília, Brasília.

REZENDE, A.V. 1998. Importância das matas de galeria: manutenção e recuperação. In Cerrado: matas de galeria (J.F. Ribeiro, ed.). EMBRAPACPAC, Planaltina, p.3-16.

RODAL, M.J.N. \& NASCIMENTO, L.M. 2002. Levantamento florístico da floresta serrana da Reserva Biológica de Serra Negra, microrregião de Itaparica, Pernambuco, Brasil. Acta Bot. Bras. 16(4):481-500.
RODAL, M.J.N., ANDRADE, K.V.A., SALES, M.F. \& GOMES, A.P.S. 1998. Fitossociologia do componente lenhoso de um refúgio vegetacional no município de Buíque, Pernambuco. Rev. Brasil. Biol. 58(3):517-526.

RODRIGUES, L.A., CARVALHO, D.A., OLIVEIRA-FILHO, A.T., BOTREL, R.T. \& SILVA, E.A. 2003. Florística e estrutura da comunidade arbórea de um fragmento florestal em Luminárias, MG. Acta Bot. Bras. 17(1):71-87.

RODRIGUES, R.R. \& NAVE, A.G. 2004. Heterogeneidade florística das matas ciliares. In Matas ciliares: conservação e recuperação (R.R. Rodrigues \& H.F. Leitão Filho, ed.). EDUSP/FAPESP, São Paulo, p.45-72.

RODRIGUES, R.R. 1989. Análise estrutural das formações florestais ripárias. In Simpósio sobre Mata Ciliar. Fundação Cargill, São Paulo, p.99-119.

ROZZA, A.F. \& RIBEIRO C.A. 1992. Estudo florístico e fitossociológico de fragmentos de mata ciliar dos Campos da ESALQ, Piracicaba, SP. In Congresso da Sociedade Botânica de São Paulo. São Paulo, p.7-12.

SAMPAIO, E.V.S. \& GAMARRA-ROJAS, C.F.L. 2003. A vegetação lenhosa das ecorregiões da Caatinga. In Desafios da botânica brasileira no novo milênio: inventário, sistematização e conservação da diversidade vegetal (E.A.G. Jardin, M.N.C. Bastos \& J.U.M. Santos, ed.). Sociedade Brasileira de Botânica, Belém, p.85-90.

SANTANA, J.A.S. 2005. Estrutura fitossociológica, produção de serrapilheira e ciclagem de nutrientes em uma área de caatinga no seridó do Rio Grande do Norte. Tese de Doutorado, Universidade Federal da Paraíba, Areia.

SANTOS, N.A. \& SOUSA-SILVA, J.C. 1998. As matas de galeria têm importância econômica? In Cerrado: matas de galeria (J.F. Ribeiro, ed.). EMBRAPA-CPAC, Planaltina, p.157-164.

SILVA JÚNIOR, M.C., FELFILI, J.M., NOGUEIRA, P.E. \& REZENDE, A.V. 1998. Análise florística de matas de galeria no Distrito Federal. In Cerrado: matas de galeria (J.F. Ribeiro, ed.). EMBRAPA-CPAC, Planaltina, p.53-84.

SILVA, F.C., FONSECA, E.P., SOARES-SILVA, L.H., MULLER, C. \& BIANCHINI, E. 1995. Composição florística e fitossociológica do componente arbóreo das florestas ciliares da bacia do rio Tibagi. 3 . fazenda Bom Sucesso, município de Saponema, PR. Acta Bot. Bras. 9(2):289-302.

SOARES-SILVA, L.H., BIANCHINI, E., FONSECA, E.P., DIAS, M.C., MEDRI, M.E. \& ZANGARO FILHO, W. 1992. Composição florística e fitossociologia do componente arbóreo das florestas ciliares da bacia do rio Tibagi. 1. Fazenda Doralice - Ibiporã, PR. Rev. Inst. Flor. 4(1):199-206.

SOUZA, J.S., ESPÍRITO-SANTO, F.D.B., FONTES, M.A.L., OLIVEIRAFILHO, A.T. \& BOTEZELLI, L. 2003. Análise das variações florísticas e estruturais da comunidade arbórea de um fragmento de floresta semidecídua às margens do rio Capivari, Lavras-MG. Rev. Árvore 27(2):185-206.

TAVARES, S., PAIVA, F.A.V., TAVARES, E.J.S. \& LIMA, J.L.S. 1974. Inventário florestal do Ceará. Estudo preliminar das matas remanescentes do município de Barbalha. Boletim de Recursos Naturais 12(2):20-46.

TAVARES, S., PAIVA, F.A.V., TAVARES, E.J.S., LIMA, J.L.S. \& CARVALHO, G.H. 1969. Inventário florestal de Pernambuco. Estudo preliminar das matas remanescentes do município de São José do Belmonte. Bol. Rec. Nat. 7(1/4):113-139.

VAN DEN BERG, E. \& OLIVEIRA-FILHO, A.T. 2000. Composição florística e estrutura fitossociológica de uma floresta ripária em Itutinga, MG, e comparação com outras áreas. Rev. Brasil. Bot. 23(3):231-253.

VEIGA, M.P., MARTINS, S.S., SILVA, I.C., TORMENA, C.A., \& SILVA, O.H. 2003. Avaliação dos aspectos florísticos de uma mata ciliar no Norte do Estado do Paraná. Acta Sci., Agron. 25(2):519-525.

VIEIRA, F.A., SANTOS, R.M., NUNES, Y.R.F. \& FAGUNDES, M. 2003. Florística e estrutura da comunidade arbórea de fragmentos de matas ciliares dos rios São Francisco, Cochá e Carinhanha, Norte de Minas Gerais, Brasil. In Congresso de Ecologia do Brasil. Fortaleza, p.330-331. 